[Review]

www.whxb.pku.edu.cn

\title{
分级结构纳米材料的液相合成策略
}

\author{
张东风牛丽亚郭林* \\ (北京航空航天大学化学与环境学院, 北京 100191)
}

\begin{abstract}
摘要：由于具有多层次、多维度、多组分的耦合和协同效应, 分级结构纳米材料的设计合成近年来吸引了广泛 的关注. 本文从分级结构的三种实现方式, 即核壳结构、节型结构和分支结构, 分别介绍其液相合成方法进展, 并 侧重从晶体成核生长和反应动力学等角度对分级结构的形成原理和影响因素进行阐述. 总的来说, 分级结构材 料的构筑主要涉及次级结构材料在初级结构材料上的异质成核生长或两种材料之间的成分交换. 其中, 材料之 间的晶格匹配度、次级结构材料的过饱和度及化学成键情况等是影响二次相成核位点及方式的重要因素, 而对 晶面能的调控是调节二次相材料生长行为的重要途径. 对基于成分交换的合成策略来讲,一个重要的前提条件 是两种材料具有相同的阴离子或阳离子.
\end{abstract}

关键词：核壳结构；节型结构；分支结构；液相合成；纳米复合材料

中图分类号： O641; 0642

\section{Solution Synthesis Strategies for Hierarchical Nanostructures}

\author{
ZHANG Dong-Feng NIU Li-Ya GUO Lin* \\ (School of Chemistry and Environment, Beihang University, Beijing 100191, P. R. China)
}

\begin{abstract}
The coupling and synergistic effects of the unique structure of multi-level, multi-dimension, and multicomponents allow for the directed synthesis of hierarchical nanostructures and this field has attracted much interest recently. In this paper, we discuss progress in the solution synthesis of three kinds of hierarchical structures including core-shell, segmented, and branched structures. We focus on the formation mechanism and the influencing factors of the hierarchical structures by considering the crystal nucleation-growth process and growth kinetics. The construction of the hierarchical nanocomposites mainly involves the heterogeneous nucleation-growth of the secondary structures on the primary structures or a component exchange between the two kinds of materials. The degree of lattice matching, the degree of supersaturation, and chemical bonding mainly influence the hetero-nucleation sites of the secondary structures on the primary structures. The growth behaviors of the secondary structures can be modulated mainly by adjusting their crystallographic energy through surface modifications. For the synthesis via component exchange, an important prerequisite is that the primary and secondary structures share the same anions or cations.
\end{abstract}

Key Words: Core-shell structure; Segmented structure; Branched structure; Solution synthesis;

Nanocomposites

现代科学技术的发展对材料提出了更高的要 求, 需要材料具备多功能、小型化、集成化等特点. 由 于具有多层次、多维度、多组分的耦合和协同效应, 分级结构材料无疑成为满足材料发展要求的最有力
的承载者. 分级结构并不是凭空臆想, 而是自然界给 人类的启示. 研究表明, 许多生物体之所以具有人工 合成材料难以逾越的神奇功能, 正是由于大自然的 巧夺天工在这些生物体中产生了高度有序的微/纳

Received: June 30, 2010; Revised: August 16, 2010; Published on Web: September 9, 2010.

*Corresponding author. Email: guolin@buaa.edu.cn; Tel: +86-10-82338162.

The project was supported by the National Natural Science Foundation of China (20803002, 50725208, 20973019) and Research Fund for the Doctoral Program of Higher Education of China (20070006016).

国家自然科学基金(20803002, 50725208, 20973019)及高校博士点新教师专项基金(20070006016)资助项目

C. Editorial office of Acta Physico-Chimica Sinica 
米多级结构. 比如, 对贝壳类珍珠质进行分析, 发现 其之所以具有高强度和高硬度, 与贝壳的片层分级 组装结构密不可分 ${ }^{[1]}$; 荷叶之所以具有超疏水特性, 很大程度上是由其表面的微/纳多级结构决定的 ${ }^{[2]}$. 科研工作者对人工构造的分级结构的研究也得到 了令人振奋的结果. 比如, 模拟生物表面不平的坎 状的多级结构, 可以实现具有选择性的表面润湿 ${ }^{[3]}$; 具有介孔壳层结构的碳空心微米球表现出很好的电

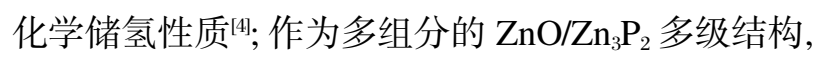
则具有作为光子检测设备的应用前景 ${ }^{[5]}$. 分级结构 材料的研究无论对于材料性能的开发, 还是对于新 型功能器件的构筑等方面都具有十分重要的意义, 分级结构材料的合成与性质研究已成为国际上的一 个前沿研究领域.

从广义上来讲, 分级结构材料既包括特殊结构的 多面体(如多足状、星状等)、零维或一维纳米粒子的二 维、三维超排列, 还包括特定形状的纳米基元通过相 互作用形成的特殊形态多级结构, 如核壳(core-shell) 结构、结型(segments)结构及分支(branches)结构等. 本文将重点从液相合成角度对特殊形态多级结构的 研究进展进行总结介绍.

\section{1 核壳结构的合成方法}

顾名思义, 核壳材料由处于中心的核以及包覆 在外部的壳组成. 当壳层数目 $\geqslant 2$ 时, 形成的结构又 称为夹层或三明治结构. 通过核壳结构的构建, 一方 面可以集几种不同的功能于一体; 另一方面壳层的 形成可以起到对核材料的表面修饰及保护作用. 比 如, 磁核-金壳结构不仅实现了光磁功能复合, 而且 金壳的存在还能有效降低磁核的生物降解性与生物 毒性, 改善粒子的生物相容性 ${ }^{[6-8]}$. 将半导体量子点 外部包覆一层光透过性好的壳, 会有效提高半导体 量子点近带边发射的量子产率, 通过调节核-壳的组 成还可以实现对材料带隙的调节 ${ }^{[9-11]}$. 较高的量子效 率以及可以调节的菼光颜色, 使半导体量子点在生 物标记领域具有巨大的应用前景 ${ }^{[12-14]}$. 然而作为生 物标记物, 除了必须具有一定的苂光亮度外, 无毒、 不易水解、不易被氧化都是几个重要的指标, 壳层的 生长正是阻隔其表面的电子、光子及氧原子扩散的 有效途径. 具有对可见光透明、易与有机功能基团结 合及较高化学稳定性等优点的 $\mathrm{SiO}_{2}$ 成为一个重要 的壳层侯选物 ${ }^{[15-18]}$.

近年来, 人们在核壳结构的制备方面取得了较
大进展, 发展了各种合成方法. 主要的合成策略如下 所述.

\section{1 模板法}

核壳结构材料制备方法中最为典型的就是模板 法. 模板法主要是利用已经存在的纳米结构如单分 散的无机物或高分子聚合物粒子作为基底, 在其表 面进行目标产物的沉积而制备核壳结构材料. 在该 方法中, 为了确保壳层材料在核上的有效吸附, 主要 采用的手段包括以下三种.

(1) 先对核粒子表面进行表面修饰, 通过目标产 物前驱体离子与表面修饰剂之间的相互作用进行壳 层粒子的沉积 ${ }^{[19-30]}$. 例如, Caruso 课题组 ${ }^{[19-21]}$ 用聚合 物作为模板, 首先通过聚电解质对合成的聚合物微 球表面进行改性, 使其表面带有一定的电荷, 然后引 发制备壳材料的反应, 通过核与壳材料之间的静电 引力使得壳材料较好地包覆在核上, 再继续对所得 到的核壳粒子进行聚电解质的吸附-反应, 达到调节 壳层厚度的目的, 如图 1 所示 ${ }^{[21]}$.

利用类似的方法, 人们合成出了多种具有核壳 结构的无机材料, 如 $\mathrm{TiO}_{2} 、 \mathrm{SiO}_{2} 、 \mathrm{CdTe}$ 与聚苯乙烯 (PS)的核壳材料等 ${ }^{[22-24]}$. 美国华盛顿大学夏幼南课 题组首先将表面修饰有聚乙烯醇(PVA)的 PS 球旋 涂在玻璃基底表面, 然后将其浸渍在含有异丙醇钛 的异丙醇溶液中, 通过异丙醇钛的水解-凝聚制备了 $\mathrm{PS} @ \mathrm{TiO}_{2}$ 核壳结构, 以甲苯溶解 PS 核获得了 $\mathrm{TiO}_{2}$ 空心蛋壳 ${ }^{[25]}$; 近来, 他们以金纳米棒为基底, 向溶液 中加人 $\mathrm{AgNO}_{3}$ 溶液, 利用聚乙烯吡咯烷酮(PVP)、十 六烷基三甲基溴化铵 $(\mathrm{CTAB})$ 与 $\mathrm{Au}$ 棒及 $\mathrm{Ag}^{+}$离子的 共同相互作用制备了十面体状 $\mathrm{Au}$ 核 @ $\mathrm{Ag}$ 壳纳米 结构 ${ }^{[26]}$. 俄克拉荷马州州立大学 Mao 等人 ${ }^{[27]}$ 先将 PVP 组装为纤维状结构, 以此为模板, 利用 $\mathrm{Si}$ 与 PVP 之间的相互作用制备了 $\mathrm{SiO}_{2}$ 空心管, 然后以 $\mathrm{SiO}_{2}$ 管为二次模板, 再次利用 PVP 与 $\mathrm{Si}$ 的相互作 用, 将表面包覆有 PVP 的 Au 纳米粒子组装在其表 面, 获得了 $\mathrm{SiO}_{2} @ \mathrm{Au}$ 核壳结构材料.

(2) 利用晶格匹配度合适的晶体间发生外延生 长的现象, 选择与核粒子具有相似晶格参数的物 质进行外延生长来实现核壳结构的制备. 锌、镉的 硫化合物具有相似的晶格匹配度, 人们基于晶体 外延生长原理制备了包括 ZnSe@CdSe、CdSe@ZnS 及 $\mathrm{CdSe} @ \mathrm{CdS}$ 等多种半导体量子点、量子线核壳结 构 ${ }^{[9-14,31-40]}$. 通过核壳结构的制备, 有效改善了量子点 的量子效率、调节了量子点的发光波长, 在多色标记 


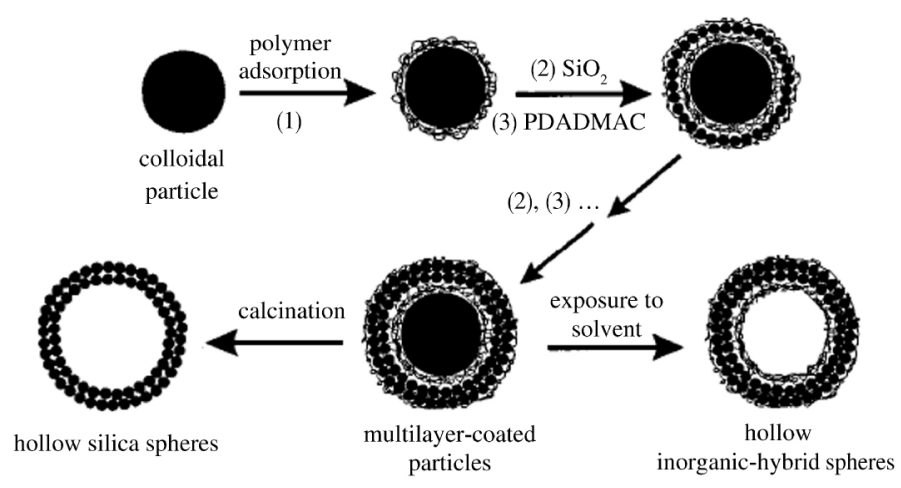

图 1 胶体球表面吸附聚电解质形成包覆反应示意图 ${ }^{[21]}$

Fig.1 Scheme illustrating the formation of core-shell structures by the adsorption of polyelectrolytes on colloidal particles ${ }^{[21]}$ PDADMAC: poly(diallyldimethylammonium chloride)

等领域具有潜在的应用前景.

近来, 晶体外延生长原理被巧妙地用于双金属 核壳结构的制备 ${ }^{[41-46]}$. 美国伯克利大学杨培东课题 组 ${ }^{[4]}$ 以预先制备好的 $\mathrm{Pt}$ 纳米立方体为晶种, 以抗坏 血酸为还原剂对 $\mathrm{KPdCl}_{4}$ 进行还原, 利用 $\mathrm{Pd}$ 在 $\mathrm{Pt}$ 粒 子表面的外延生长制备了核壳结构的 Pt@Pd 多面 体, 由于 $\mathrm{Pt}$ 与 $\mathrm{Pd}$ 之间近乎完美的晶格匹配度, 所制 备的 Pd 壳具有单晶结构, 而且通过 $\mathrm{NO}_{2}$ 在 $\mathrm{Pd}$ 粒子 表面的晶面选择性吸附, 实现了产物的形状控制, 如 图 2 所示.

厦门大学田中群课题组 ${ }^{[42]}$ 以 $\mathrm{Au}$ 八面体纳米粒 子为基底, 在表面活性剂 CTAB 的存在下, 以抗坏血 酸为还原剂制备了尺寸均一的 $\mathrm{Au} @ \mathrm{Pd} 、 \mathrm{Au} @ \mathrm{Ag}$ 核 壳结构立方块. 该方法还被用于构筑 $\mathrm{Au} @ \mathrm{Pt}^{[44]}$ 、 $\mathrm{Pt} @ \mathrm{Ru}^{[45]} 、 \mathrm{Au} @ \mathrm{Ag}^{[46]}$ 等双金属体系的核壳结构.

(3) 通过调控过饱和度来实现目标粒子在基底 的沉积, 从而实现核壳结构的制备. 无论是对基底粒 子进行表面修饰, 还是选择与基底具有合适晶格匹 配度的壳层都会有效降低异相成核生长的界面能. 根据晶体成核-生长理论, 过饱和度是决定粒子沉积 方式的另一个重要因素. 因此通过控制反应参数如 反应温度、反应物浓度、前驱体种类、 $\mathrm{pH}$ 环境等因 素, 使目标粒子处于合适的过饱和度, 可以促进粒子 在基底的异相成核生长, 从而实现核壳结构的制备. 中国科学院物理研究所王太红等人 ${ }^{[47]}$ 以 $\mathrm{ZnO}$ 纳米 线为模板, 通过控制反应体系的酸碱性, 对原硅酸四 乙酯(TEOS)的水解凝聚速率进行控制, 实现了 $\mathrm{SiO}_{2}$ 在 $\mathrm{ZnO}$ 表面的沉积. Krishna 等人 ${ }^{[48]}$ 采用同样的思 路, 以 $\mathrm{ZnO}$ 微米鼓为基底, 进行原位沉积制备了 $\mathrm{ZnO} @ \mathrm{SiO}_{2} 、 \mathrm{ZnO} @ \mathrm{Au} 、 \mathrm{ZnO} @ \mathrm{C}$ 等鼓状核壳复合结
构. Teng 等人 ${ }^{[49]}$ 通过在辛醚中还原乙酰丙酮铂制备 了 Pt 纳米粒子, 以它为模板通过化学沉积的方法在 其表面包覆了一层 $\mathrm{Fe}_{2} \mathrm{O}_{3}$ 纳米粒子, 得到了 $\mathrm{Pt} / \mathrm{Fe}_{2} \mathrm{O}_{3}$ 核壳材料. 清华大学李亚栋课题组 ${ }^{[50]}$ 以单分散的 C 纳米球作为模板, 通过水解 $\mathrm{WCl}_{6}$ 得到了 $\mathrm{C} / \mathrm{WO}_{3}$ 核 壳材料, 通过高温陆烧除去 $\mathrm{C}$ 模板, 得到了直径在 $400 \mathrm{~nm}$ 、壳厚为 $30 \mathrm{~nm}$ 的 $\mathrm{WO}_{3}$ 中空纳米球. 研究发 现, 该球对乙醇、丙唒、二硫化碳等气体具有一定的 灵敏度, 适合于做气体传感器.

此外, 相界面也常被人们巧妙地用于层壳结构 的构筑. 比如, 徐正课题组 ${ }^{[51]}$ 在环己胺/聚乙二醇/水 组成的乳液体系中, 通过 $\mathrm{NiSO}_{4}$ 和 $\mathrm{NaH}_{2} \mathrm{PO}_{2}$ 两者氧 化还原反应制备了直径在 $300 \mathrm{~nm}$ 左右的中空 $\mathrm{Ni}$ 球. 在乳液体系中, 由于环己胺不溶于水, 在水相里 以油滴形式存在, 而聚乙二醇既有疏水基又有亲水 基, 它在油水界面上起到桥梁的作用, 稳定了水包油 液滴, 聚乙二醇链上的氧原子对 $\mathrm{Ni}^{2+}$ 有较强的亲合
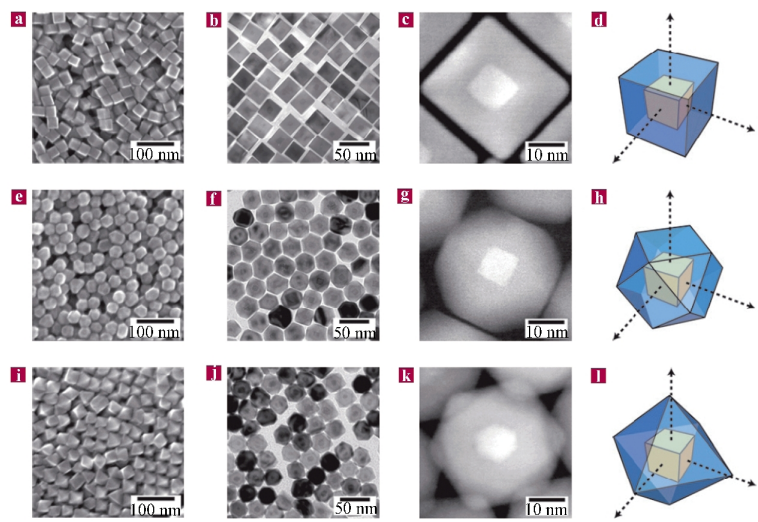

图 2 以晶体外延生长法制备的 Pt@Pd 多面体 ${ }^{[41]}$

Fig.2Pt@Pd nanopolyhedrons synthesized via crystallographic epitaxial growth ${ }^{[4]}$ 
力, 使得溶液中的 $\mathrm{Ni}^{2+}$ 被吸附在乳液液滴的界面上, 导致了 $\mathrm{NiSO}_{4}$ 和 $\mathrm{NaH}_{2} \mathrm{PO}_{2}$ 氧化还原发生在界面上, 生成了以乳液液滴为模板的 $\mathrm{Ni}$ 金属层 ${ }^{[5]}$.

\section{2 柯肯达尔效应法}

柯肯达尔(Kinkendall)效应是指两种扩散速率 不同的组分在互扩散过程中会在分界面处形成空隙 缺陷. 近年来, 柯肯达尔效应已成为制备核壳结构的 一种重要方法. 在反应过程中, 作为核的粒子既作为 壳材料形成的模板, 又作为一种反应物参与了壳材 料的生成反应. 在壳材料围绕模板生长的同时, 模板 自身被不断消耗. 因此该方法是一种牺牲模板法. 通 过控制反应进程, 可以对核壳的相对尺寸进行有效 调控.

柯肯达尔效应被用于核壳结构纳米粒子的合成 始于 2004 年 Alivisatos 课题组 ${ }^{[52]}$ 的工作. 他们以分 散在邻氯甲苯中 Co 纳米粒子作为牺牲模板, 通过 向其中注射 $\mathrm{S}$ 或 $\mathrm{Se}$ 的邻氯甲苯溶液, 分别制备了 $\mathrm{Co} @ \mathrm{CoS}$ 及 $\mathrm{Co} @ \mathrm{CoSe}$ 核壳结构, Co 核被快速耗尽, 从而形成了 $\mathrm{CoS}$ 及 $\mathrm{CoSe}$ 空心结构, 如图 3 所示. 将 $\mathrm{Co}$ 核在 $\mathrm{Ar} / \mathrm{O}_{2}$ 混合气氛中处理, 则获得了 $\mathrm{Co} @ \mathrm{CoO}$ 结构 ${ }^{[52]}$. 另外他们还以 $\mathrm{Pt} @ \mathrm{Co}$ 核壳结构粒子为牺牲 模板, 利用柯肯达尔效应制备了 $\mathrm{Pt} @ \mathrm{CoO}$ 荚状核壳 结构 ${ }^{[53]}$. 自 Alivisatos 组的工作在 Science 杂志发表 以来, 该方法被迅速引用 ${ }^{[54-63]}$. 比如, 香港科技大学 徐兵课题组 ${ }^{[54]}$ 通过向新制备的 FePt@Co 核壳结构 纳米粒子溶液中注入硫的邻氯甲苯溶液中, 制备了 $\mathrm{FePt} @ \mathrm{CoS}_{2}$ 荚状核壳结构.

夏幼南研究组 ${ }^{[5]}$ 以直径约为 $200 \mathrm{~nm}$ 的单分散 的铅纳米球为模板, 利用 Kirkendall 效应及铅的较 高反应活性, 在较低的温度 $\left(300{ }^{\circ} \mathrm{C}\right)$ 与硫粉反应, 得
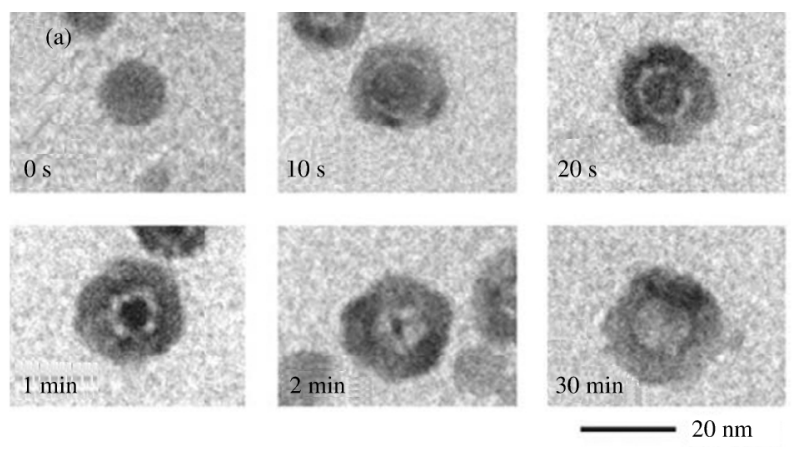

图 3 基于柯肯达尔效应, 从 $\mathrm{Co}$ 纳米粒子到 CoSe 空心结 构形貌演变过程 ${ }^{[2]}$

Fig.3 Morphology evolvement from Co nanoparticles to CoSe hollow structures through Kirkendall effect ${ }^{[52]}$
到了空心的 $\mathrm{Pb} @ \mathrm{PbS}$ 核壳纳米球以及硫化铅空心 球, 在硝酸银溶液中通过取代反应还可得到 $\mathrm{Pb} @ \mathrm{Ag}$ 核壳结构的粒子.

北京大学徐东升课题组 ${ }^{[5]}$ 以 $\mathrm{Cu}_{2} \mathrm{O}$ 为牺牲模板 制得了 $\mathrm{Cu}_{x} \mathrm{~S}$ 空壳结构. 实验的关键在于 $\mathrm{Cu}_{2} \mathrm{O}$ 晶格 中 $\mathrm{O}$ 原子和溶液中 $\mathrm{S}$ 原子的置换作用. 由于 $\mathrm{S}$ 原子 和 $\mathrm{O}$ 原子在反应过程中的扩散速度不同, 使得 $\mathrm{Cu}_{2} \mathrm{O} / \mathrm{Cu}_{x} \mathrm{~S}$ 核壳结构的壳层存在裂隙, 可以用氨水 溶解 $\mathrm{Cu}_{2} \mathrm{O}$ 核从而得到 $\mathrm{Cu}_{x} \mathrm{~S}$ 空壳结构, 进一步研究 表明, 可以通过改变实验过程中通人的气体来控制 产物中 $\mathrm{Cu}$ 和 $\mathrm{S}$ 的原子个数比.

由于核粒子的结构模板作用, 还可以通过核粒 子形状的选择制备具有不同构型的核壳结构粒子. 比如徐兵课题组 ${ }^{[57]}$ 以链状 Co 纳米粒子作为牺牲模 板制备了豆荚状 Co@ CoSe 核壳结构. Fan 等人 ${ }^{[58-59]}$ 以 $\mathrm{MgO}$ 带作为牺牲模板制备了同样具有带状结构 的 $\mathrm{MgO} @ \mathrm{MgAl}_{2} \mathrm{O}_{4}$ 核壳.

最近, Tracy 等人基于柯肯达尔效应将 $\mathrm{Ni}$ 纳米 粒子转化为 $\mathrm{NiO}$ 空心纳米球. 他们的研究表明, $\mathrm{Ni}$ 粒子的尺寸离子会影响离子的扩散途径, 进而对产 物 $\mathrm{NiO}$ 空心纳米球的微结构造成较大影响[00].

\section{3 氧化还原反应法}

根据氧化还原原理, 若 $\varphi\left(\mathrm{A}^{n+} / \mathrm{A}\right)>\varphi\left(\mathrm{B}^{m+} / \mathrm{B}\right)$, 则会 发生反应: $m \mathrm{~A}^{n+}+n \mathrm{~B} \rightleftharpoons m \mathrm{~A}+n \mathrm{~B}^{m+}$. 因此向分散有 $\mathrm{B}$ 纳 米粒子的溶液中, 加人 $\mathrm{A}^{n+}$ 离子, 则通过控制反应条 件会生成以 $\mathrm{B}$ 为核, $\mathrm{A}$ 为壳的纳米复合粒子. 这一 原理已被广泛用于双金属核壳结构及空心材料的制 备. 夏幼南课题组在此方面做出了具有开创性的工 作. 以预先制备好的 $\mathrm{Ag}$ 纳米立方体作为基底, 通过 向其中加人电极电势较高的其他金属离子如 $\mathrm{AuCl}_{4}^{-}$, $\mathrm{PtCl}_{6}^{2-}, \mathrm{PdCl}_{6}^{2-}$ 等, 成功制备了 $\mathrm{Au}-\mathrm{Ag}, \mathrm{Pt}-\mathrm{Ag}, \mathrm{Pd}-\mathrm{Ag}$ 等双金属空心结构纳米粒子[64-68]. 通过 PVP 等表面 活性剂对 $\mathrm{Ag}$ 粒子的晶面选择性吸附, 还可以进一 步调节氧化还原发生的位点, 从而达到对空心材料 微结构进行调控的目的 ${ }^{[6]}$, 如图 4 所示. 通过向空 心结构多次进行沉积-置换, 可以制备多壳层纳米 结构 ${ }^{[70-71]}$.

Lee 课题组 ${ }^{[72-73]}$ 利用氧化还原反应过程制备了 一系列以 Co 为核的核壳结构纳米颗粒. 天津大学 师春生等人 ${ }^{[74]}$ 在水/正辛烷体系中, Brij30 作为表面 活性剂, 用 $\mathrm{NaBH}_{4}$ 还原 $\mathrm{NiCl}_{2}$ 生成 $\mathrm{Ni}$ 核, 再加人 $\mathrm{HAuCl}_{4}$ 的微乳液, 利用 $\mathrm{Ni} / \mathrm{HAuCl}_{4}$ 之间的氧化还原 反应制备了核壳结构的 $\mathrm{Ni} @ \mathrm{Au}$. 中国科学技术大学 


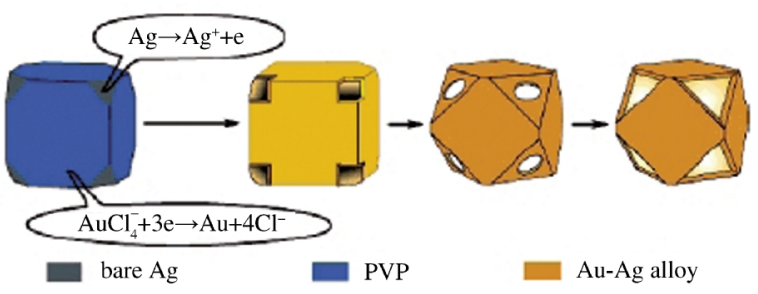

图 4 PVP 保护作用下, 由 Ag 纳米立方体到 Au-Ag 空心 笼的转变过程示意图 ${ }^{[69]}$

Fig.4 Scheme illustrating the transformation from PVP-protected Ag nanocubes to Au-Ag hollow nanocages $^{[69]}$

PVP: polyvinylpyrrolidone

俞书宏课题组 ${ }^{[75]}$ 在 PVP 的存在下, 利用 Co、 $A u$ 具有 的不同还原电势, 以 $\mathrm{AuCl}_{4}^{-}$还原 $\mathrm{Co}$ 纳米粒子, 制备 了 $\mathrm{Co} @ \mathrm{Au}$ 核壳结构粒子. 该方法的优势在于核壳 结构的形成不需要填加额外的还原剂, 从而降低体 系的复杂度, 减少污染离子.

\section{2 节型结构}

节型结构指两种或多种组分纳米粒子通过首尾 顺次连接形成的片段型一维结构. 这种结构不仅集 多组分的功能于一身, 而且异质结的存在还使其在 光发射、光传导、光检测、生物传感、基因转移及催化 等多个领域具有潜在应用价值 ${ }^{[76-84]}$. 近年来, 液相法 在合成节型结构方面也表现出极大的发展潜力.

\section{1 价键诱导法}

节型结构的形成涉及到第二相物质成核位点的 精准控制, 这也是节型结构制备过程中面临的一大 挑战. 在无外力作用的情况下, 一般需要在表面修饰 剂的保护下, 通过动力学反应过程的调控来实现.

2004 年, Banin 等人 ${ }^{[85]}$ 将 $\mathrm{AuCl}_{3}$ 溶人加有双十二 烷基二甲基溴化铵(DDAB) 和十二烷胺的甲苯溶液 中, 与预先制备好的 $\mathrm{CdSe}$ 溶胶混合, 使得 $\mathrm{Au}$ 纳米 颗粒在 $\mathrm{CdSe}$ 纳米棒顶端进行选择性生长. 通过改 变反应物的浓度可以控制 $\mathrm{Au}$ 颗粒的大小. 这种选 择性生长一方面是由于配合物在纳米棒端面的不完 全钝化, 使得端面的表面能较高, 另一方面他们认为 在纳米棒的端面形成了 $\mathrm{Au}-\mathrm{Se}$ 键, 诱导了 $\mathrm{Au}$ 在端 面的生长.

基于共价键的诱导生长在他们之后的研究中得 到了进一步的印证 ${ }^{[86-87]}$. 在相似的条件下, 将溶有 $\mathrm{AuCl}_{3}$ 、DDAB 和十二烷胺的甲苯溶液滴加到分散有 $\mathrm{CdS}$ 纳米棒的甲苯溶胶混合, 成功制备了 $\mathrm{Au}-\mathrm{CdS}$ 节型结构. 为了探究生长机理, 反应分别在空气和
$\mathrm{Ar}$ 气中进行. 在空气中进行时, 金粒子可以在 $\mathrm{CdS}$ 纳米棒的两端生长, 而在 $\mathrm{Ar}$ 气中, $\mathrm{Au}$ 粒子只在棒的 一端生长. 他们认为, $\mathrm{CdS}$ 纳米棒的一端以 $\mathrm{Cd}$ 原子 为终结面, 另一端以 $\mathrm{S}$ 原子为终结面, 在 $\mathrm{Ar}$ 气中, $\mathrm{Au}-\mathrm{S}$ 键只在一端形成, 从而导致 $\mathrm{Au}$ 粒子在棒的 一端生长; 在空气中, 由于 $\mathrm{O}$ 对 $\mathrm{Cd}$ 原子的刻蚀, 使 $\mathrm{S}$ 原子暴露, 因此在棒的两端都有 $\mathrm{Au}-\mathrm{S}$ 键的形成, 从而导致了 $\mathrm{Au}-\mathrm{CdS}-\mathrm{Au}$ 结构的形成, 如图 5 所示. 利用奥氏熟化机理, 还可以对结构进行进一步调控, 使 $\mathrm{Au}$ 纳米颗粒只在 $\mathrm{CdSe}$ 棒的一端生长 ${ }^{[88]}$.

孙守恒等人 ${ }^{[89]}$ 将 $\mathrm{Au}$ 纳米颗粒和加有油酸油胺 的 $\mathrm{Fe}(\mathrm{CO})_{5}$ 十八烷烯溶液混合并加热回流, $\mathrm{Fe}(\mathrm{CO})_{5}$ 在 $\mathrm{Au}$ 颗粒表面沉积并被空气中的氧气氧化成 $\mathrm{Fe}_{3} \mathrm{O}_{4}$, 在 $\mathrm{Au}$ 颗粒表面进行外延生长.

在同样的原理指导下, $\mathrm{Au}-\mathrm{PbS} 、 \mathrm{Au}-\mathrm{PbSe} 、 \mathrm{PbSe}-$ $\mathrm{CdSe}-\mathrm{PbSe} 、 \mathrm{PbSe}-\mathrm{CdS}-\mathrm{PbSe} 、 \mathrm{CdS}-\mathrm{Ag}_{2} \mathrm{~S}$ 等节型结构 被成功合成 ${ }^{[00-95]}$.

\section{2 多孔模板与电化学沉积}

利用多孔物质(尤其是多孔氧化铝及多孔聚合 物膜)的空间限域效应, 制备一维纳米材料已成为一 种成熟的方法. 这种方法简单而有效, 不管目标物质 具有什么样的结构及相态, 只要能有效地填人模板 的孔道中, 就可以得到相应的一维结构. 纳米材料的 尺寸和长度可以通过调节模板的孔径和深度方便地 得到调节. 最初人们通过简单的气相沉积或化学沉 积的方法, 借助孔的毛细作用力将目标物质填充到 多孔模板的孔道中 ${ }^{[96-99]}$. 但这容易造成填充效率不 高, 使产物在干燥的过程中出现收缩、断裂等现象. 为了使目标产物有效地进人孔道, Limmer 等人 ${ }^{[100]}$ 又发展出了电解法和溶胶一凝胶法相结合的方法, 以 基底作为阳极, 制备了致密的 PZT 纳米棒. 此后, 多

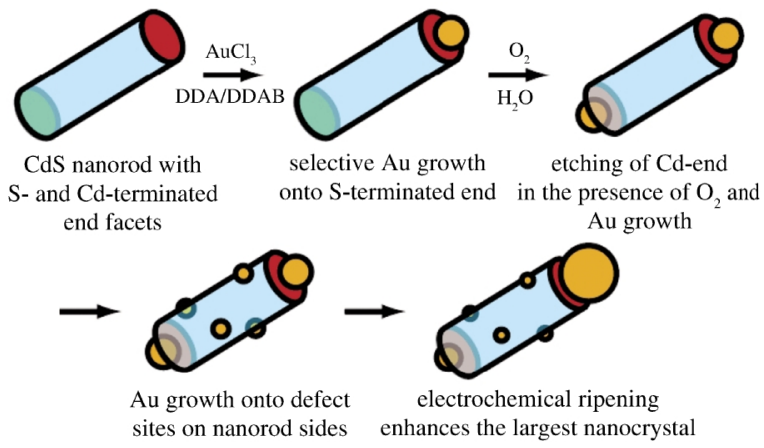

图 5 基于共价键诱导的 $\mathrm{Au}-\mathrm{CdS}-\mathrm{Au}$ 结型结构形成 示意图 ${ }^{[8]}$

Fig.5 Scheme illustrating the formation of Au-CdSAu hierarchical structures ${ }^{[8]}$ 
孔模板与电化学沉积相结合的方法被有效用于多种 一维纳米结构的制备.

显然, 若将不同物质在多孔模板中顺次沉积, 则可以得到结型结构纳米材料. 近年来, 该方法在 节型结构的制备方面取得了较大进展. 比如, Natan 等人 ${ }^{[101]}$ 首先向多孔氧化铝底部沉积一层金属 $\mathrm{Ag}$ 膜, 以此为工作电极, 通过向溶液中顺次加人不同的金 属离子, 金属离子的电化学沉积产生了包括 $\mathrm{Au}-\mathrm{Ag}-$ $\mathrm{Au}-\mathrm{Ag}$ 及 $\mathrm{Au}-\mathrm{Ag}-\mathrm{Ni}-\mathrm{Pd}-\mathrm{Pt}$ 等多金属结型棒状结构. 中国科学院化学研究所万立骏课题组 ${ }^{[102]}$ 用直径 20 $\mathrm{nm}$ 的多孔阳极氧化铝 (AAO)作为模板, 首先利用循 环伏安法找到 $\mathrm{Ni}$ 和 $\mathrm{Pt}$ 的沉积电压, 然后在三电极 电化学系统中利用脉冲电化学沉积法得到了 Pt-Ni 节型结构, 如图 6 所示.

利用类似的方法, 人们已成功制备了包括 $\mathrm{Bi}$ $\mathrm{Se} 、 \mathrm{Au}-\mathrm{CdSe}-\mathrm{Au} 、 \mathrm{Au}-\mathrm{Ni}-\mathrm{Au} 、 \mathrm{Au}-\mathrm{Ag}$ 等多种体系的节 型结构 ${ }^{[103-106]}$.

\section{3 离子交换}

Alivisatos 等人 ${ }^{[107]}$ 发现向 $\mathrm{CdE}(\mathrm{E}=\mathrm{S}, \mathrm{Se}, \mathrm{Te})$ 半 导体纳米粒子中加人 $\mathrm{Ag}^{+}$离子, 则 $\mathrm{Ag}^{+}$可以取代 $\mathrm{Cd}^{2+}$ 从而原位形成 $\mathrm{Ag}_{2} \mathrm{E}$ 纳米粒子. 于是他们想到, 若可 以控制离子交换的速度, 则通过部分阳离子交换可 以获得 $\mathrm{CdE}-\mathrm{Ag}_{2} \mathrm{E}$ 异质结构材料 ${ }^{[108]}$. 他们将表面光 滑、直径均匀的 $\mathrm{CdS}$ 纳米棒加人由 $\mathrm{AgNO}_{3}$ 、甲苯和 甲醇组成的混合溶液中, 通过控制 $\mathrm{Ag}^{+} / \mathrm{Cd}$ 比例, 在 $-66{ }^{\circ} \mathrm{C}$ 的温度下反应, 最终得到了 $\mathrm{CdS} 、 \mathrm{Ag}_{2} \mathrm{~S}$ 交替 排列的超晶格异质结构, 如图 7 所示.

后来, 他们对周期性结构的形成机理进行了深 人研究, 从晶格应力及扩散控制生长的角度进行了 解释. 认为首先 $\mathrm{Ag}^{+}$吸附在纳米棒表面形成小尺寸 的 $\mathrm{Ag}_{2} \mathrm{~S}, \mathrm{CdS}-\mathrm{Ag}_{2} \mathrm{~S}$ 界面与主体间存在正的界面形 成能, 阳离子的迅速扩散导致最初形成的 $\mathrm{Ag}_{2} \mathrm{~S}$ 发生 奥氏熟化. 当 $\mathrm{Ag}_{2} \mathrm{~S}$ 长到纳米棒直径大小时, 由于
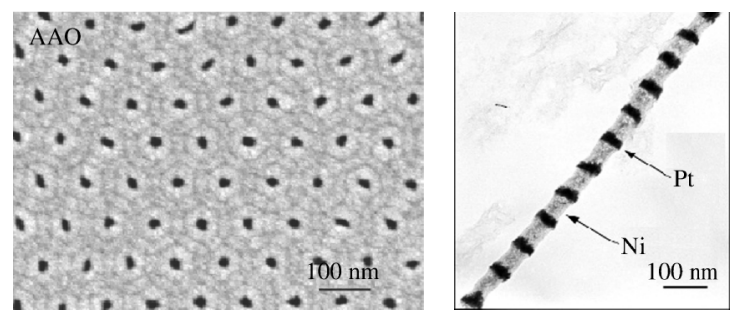

图 6 以多孔氧化铝(AAO)为模板, 通过脉冲电沉积制备的 Pt-Ni 结型异质结构 ${ }^{[102]}$

Fig.6 Pt-Ni segments fabricated using a porous anodic aluminum oxide (AAO) template by pulse electrodeposition $^{[102]}$
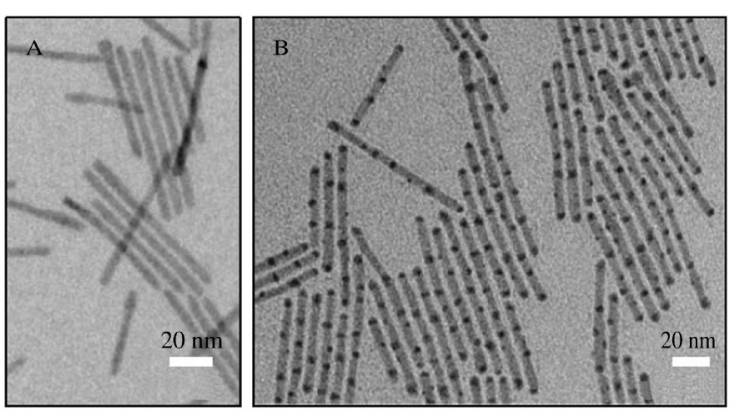

图 7 (a) CdS 纳米棒前体和(b)通过离子交换制备的 CdS/ $\mathrm{Ag}_{2} \mathrm{~S}$ 超晶格异质结构纳米棒的透射电镜照片 ${ }^{[108]}$

Fig.7 Transmission electron microscopy (TEM) images of (a) original CdS nanords and (b) $\mathrm{CdS} / \mathrm{Ag}_{2} \mathrm{~S}$ superlattices formed through cation exchange ${ }^{[108]}$

$\mathrm{Ag}^{+}$的原子间交换不会减少总的界面能, 奥氏熟化停 止; $\mathrm{CdS}$ 与 $\mathrm{Ag}_{2} \mathrm{~S}$ 之间存在的晶格失配产生的应力使 $\mathrm{Ag}_{2} \mathrm{~S}$ 片段间存在弹性排斥. 当两个 $\mathrm{Ag}_{2} \mathrm{~S}$ 片段相互 接近时, 储存在纳米棒中的弹性力大大增加, 熟化过 程停止, 从而形成了这种周期性的结构 ${ }^{[109]}$.

自 Alivisatos 课题组发现离子交换现象以来, 以 一定形状的纳米结构(尤其是 $\mathrm{CdE}$ 纳米粒子)为牺牲 模板, 通过阳离子交换已有多种纳米粒子包括 $\mathrm{PbS}$ 、 $\mathrm{Cu}_{2} \mathrm{~S}$ 等被原位合成 ${ }^{[110-112] . ~}$ 然而, 关于节型结构的报 道却相对较少, 这可能与离子交换相对较快, 难以控 制有关.

\section{3 分支结构}

通俗地讲, 分支结构指的是在主干结构上组装 有二级或多级结构的复杂结构材料. 由于次级结构 材料的成核位点及生长方式与多种实验变量如表面 活性剂、反应物浓度、反应温度、溶液 $\mathrm{pH}$ 值等密切 相关, 因而分支结构材料具有构造丰富可调的特点, 也为原器件的设计奠定了基础.

从组装原理的角度来讲, 构建纳米材料分支结 构的方法可以分为以下几种.

\section{1 晶体二次成核生长}

液相化学法合成纳米材料的反应过程中涉及从 溶液相中析出固体相的过程. 根据晶体成核-生长理 论, 过饱和度是决定粒子沉积方式的一个重要因素. 当溶液中存在固体表面时, 达到一定过饱和度的溶 液热力学不稳定, 自由能较高, 溶液中溶剂分子结合 在一起以降低体系自由能, 溶质会在固体表面优先 析出, 即发生成核过程(产生晶种). 利用该现象, 人 们将具有特定形状的纳米结构引入溶液作为基底, 通过生长动力学过程调控, 获得了多种分支结构纳 
米材料.

$\mathrm{Gao}$ 等人 ${ }^{[113]}$ 首先制得了尺寸均匀的 $\mathrm{ZnO}$ 六方 对称性纳米棒, 然后将其作为基底分散在 $\mathrm{Zn}(\mathrm{OH})_{4}^{2-}$ 饱和溶液中, 通过在 $100{ }^{\circ} \mathrm{C}$ 下水热反应得到了在纳 米棒的六个侧面组装有二次纳米棒阵列的转子状 $\mathrm{ZnO}$ 分支结构, 如图 5 所示. 实验结果证实, $\mathrm{Zn}(\mathrm{OH})_{4}^{2-}$ 的浓度是决定二次结构生长与否的关键因素. 作者 基于对 $\mathrm{ZnO}$ 成核生长行为的理解, 通过对 $\mathrm{Zn}(\mathrm{OH})_{4}^{2-}$ 离子浓度及反应温度进行简单调节, 合成了在纳米 花花瓣上组装有纳米棒阵列的 $\mathrm{ZnO}$ 分支状复杂结 构材料 ${ }^{[114]}$.

次级结构在基底表面的成核位点显然与基底各 暴露面的晶面能大小直接相关. 引人表面活性剂或 有机分子, 通过与基底特定晶面的相互作用可以实 现基底材料表面能的调节, 从而达到定向成核生长 的目的. 同样的, 通过功能分子的晶面选择性吸附, 还可以进一步对次级结构的生长方式进行调控.

比如, $\operatorname{Tian}^{[115]}$ 和 $\mathrm{Liu}^{[1150-117]}$ 等人分别利用丙二 胺(DAP) 与柠檬酸根离子对 $\mathrm{ZnO}$ 的晶面选择性吸 附, 一方面对次级结构的成核位点进行调控, 另一 方面对次级结构的生长方式进行调控, 通过多次成 核生长, 获得了构型丰富的 $\mathrm{ZnO}$ 分支结构材料. 研 究结果初步表明, DAP 倾向于在 $\mathrm{ZnO}$ 纳米棒的柱 面吸附, 而柠檬酸根倾向于端面吸附. 当溶液中存在 适量的 DAP 时, DAP 在基底 $\mathrm{ZnO}$ 柱面的吸附导致

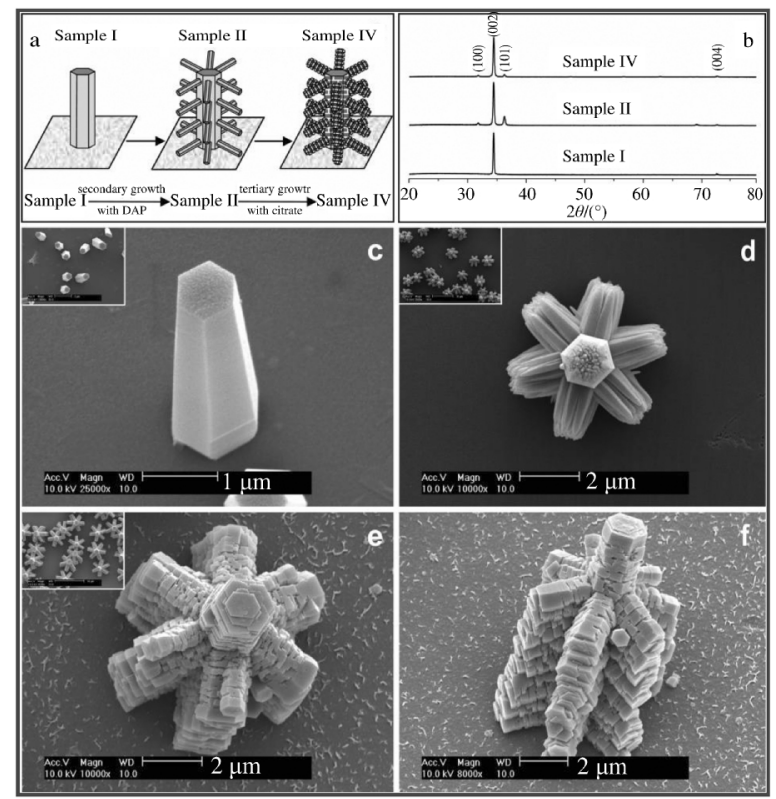

周围较高的 $\mathrm{OH}^{-}$离子浓度, 静电相互作用使得溶液 中的 $\mathrm{Zn}^{2+}$ 靠近柱面进而发生沉积, 因此获得在 $\mathrm{ZnO}$ 柱面组装有 $\mathrm{ZnO}$ 纳米棒阵列的分支结构材料; 当 DAP 浓度很高时, 基底 $\mathrm{ZnO}$ 柱面完全被 DAP 覆盖, 表面能大大降低, 抑制了 $\mathrm{ZnO}$ 次级结构的沉积, 产 物为 $\mathrm{ZnO}$ 棒. 以柠檬酸分子作为吸附剂时, 柠檬酸 根离子在 $\mathrm{ZnO}$ 端面的选择性吸附, 有效降低了端面 的表面能,一方面使得次级结构在基底能量较高的 柱面发生沉积, 另一方面降低了次级结构 $\mathrm{ZnO}$ 纳米 棒沿着 $c$ 轴的生长速度, 获得在基底 $\mathrm{ZnO}$ 棒侧面组 装有 $\mathrm{ZnO}$ 片的复合结构. 如图 8 所示 ${ }^{[115]}$.

\section{2 晶体外延生长}

虽然气相外延生长已成为一种制作二维量子阴 结构的成熟方法, 但晶体外延生长原理用于微/纳多 级结构的制备却仅仅开始于最近几年. 2002 年, 波 士顿大学的 Ren 课题组 ${ }^{[118]}$ 在研究中发现, 将 $\mathrm{ZnO}$, $\mathrm{In}_{2} \mathrm{O}_{3}$ 和石墨粉混合, 经过高温气相反应会形成以 $\mathrm{In}_{2} \mathrm{O}_{3}$ 纳米线为基底的 $\mathrm{ZnO} / \mathrm{In}_{2} \mathrm{O}_{3}$ 有序组装结构, 组 装体的构造取决于 $\mathrm{In}_{2} \mathrm{O}_{3}$ 纳米线截面的形状. 2004 年, 瑞典科学家 Samuelson 课题组 ${ }^{[119]}$ 在气-液-固(VL-S)思想指导下, 以金颗粒作为催化剂, 在阵列化的 $\mathrm{GaP}$ 纳米线 “树干”上通过外延生长获得了 $\mathrm{GaP} / \mathrm{GaP}$ 及 $\mathrm{GaP} / \mathrm{GaAsP}$ “树”状阵列.

与气相法相比, 液相法由于具有更为丰富可调 的实验参数, 可以极大提高二次相的成核位点及晶

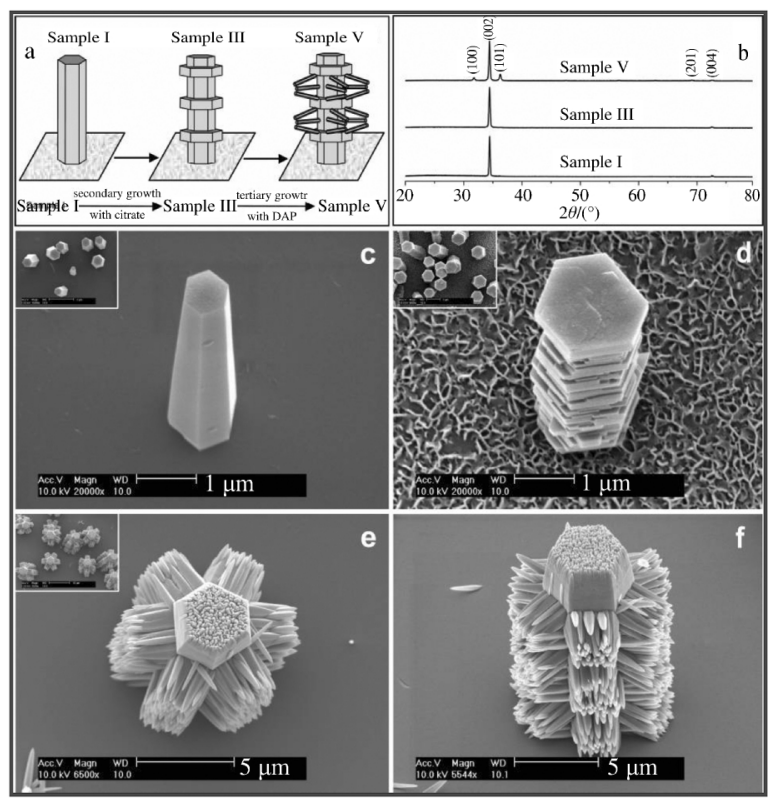

图 8 利用丙二胺和柠檬酸根离子对 $\mathrm{ZnO}$ 的晶面选择性吸附, 对 $\mathrm{ZnO}$ 多级结构的构造进行调控 ${ }^{[115]}$

Fig.8 Modulation of the growth behaviors of $\mathrm{ZnO}$ hierarchical structures using the crystallographic selective adsorption of propyldiamine and citrate anions ${ }^{[15]}$ 
体生长方式的可控性, 从而使微/纳米多级结构的设 计更具有目标性. 2004 年, 美国科学家 Alivisatos 及 其同事们 ${ }^{[120]}$ 通过精确控制晶体生长的动力学过程, 巧妙地利用晶体外延生长原理, 首先在 $\mathrm{CdS}$ 纳米棒 的顶端外延生长 $\mathrm{CdSe}$ 量子点, 接着以 $\mathrm{CdSe}$ 为核, 外延生长了四足状的 $\mathrm{CdTe}$, 最后在四足的每个分支 末端进行再一次的四足状外延生长, 在溶液中成功 制备了 $\mathrm{CdE}(\mathrm{E}=\mathrm{S}, \mathrm{Se}, \mathrm{Te})$ 纳米多级异质结构.

新加坡国立大学 Zeng 等人 ${ }^{[121]}$ 合成的 $\alpha-\mathrm{MoO}_{3} /$ $\mathrm{TiO}_{2}$ 分级结构中, $\alpha-\mathrm{MoO}_{3}$ 的(010)面和 $\mathrm{TiO}_{2}$ 的(001) 面具有相似的晶格常数, 使得在这两个界面间发生 晶体的外延生长成为可能. 同样, 他们还成功制备了 $\mathrm{ZnO} / \mathrm{TiO}_{2} / \mathrm{H}_{2} \mathrm{Ti}_{5} \mathrm{O}_{11}$ 和 $\mathrm{ZnO} / \mathrm{TiO}_{2}$ 分支结构 ${ }^{[122]}$.

我们利用外延生长原理, 成功获得在 $\alpha-\mathrm{Fe}_{2} \mathrm{O}_{3}$ 纳米管六个侧面组装有 $\mathrm{SnO}_{2}$ 纳米棒阵列的六方对 称性 $\mathrm{SnO}_{2} / \alpha-\mathrm{Fe}_{2} \mathrm{O}_{3}$ 分支结构纳米材料. 结构分析结 果表明, 与自由生长状态相比, 复合体的形成对次级 结构纳米基元 $\left(\mathrm{SnO}_{2}\right.$ 纳米棒)的生长方向从本征的 [001]方向转变为[101]方向. 进一步的分析表明, 降 低的晶格失配度是导致 $\mathrm{SnO}_{2}$ 生长方式发生变化的 驱动力 ${ }^{[123], ~}$ 如图 9 所示.

\section{3 结构导向生长}

一些有机分子、聚合物分子、生物分子及其小分 子可以对纳米粒子的成核、生长及组装过程产生调
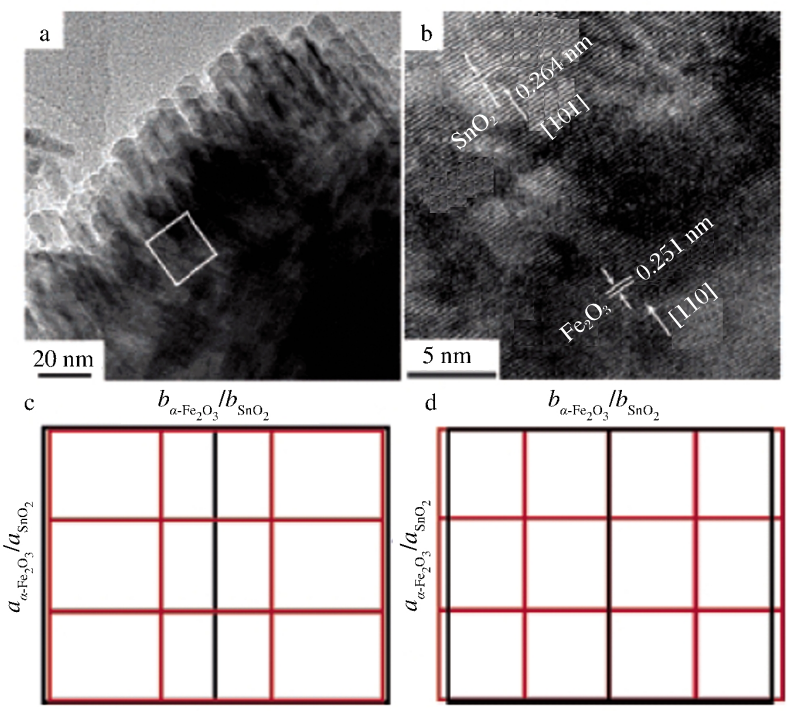

图 $9 \mathrm{SnO}_{2} / \alpha-\mathrm{Fe}_{2} \mathrm{O}_{3}$ 分支结构纳米材料界面处 TEM 照片 $(a, b)$ 和晶格匹配示意图 $(c, d)^{[123]}$

Fig.9 TEM images $(a, b)$ and schematics of the interfacial lattice match (c, d) of $\mathrm{SnO}_{2} / \alpha-\mathrm{Fe}_{2} \mathrm{O}_{3}$ branched structures ${ }^{[123]}$
控或导向作用, 起到“动态软模板”的作用. 软模板法 在复杂形态多级结构的构筑方面取得了不少成功的 实例. 从材料体系上来讲, 软模板法制备的材料主要 集中在单组分材料方面.

北京大学齐利民教授课题组在此方面作出了一 系列成果. 他们利用聚合物的诱导作用在反胶束介 质中合成了羽毛状的 $\mathrm{BaWO}_{4}$ 纳米线超结构 ${ }^{[124]}$; 通 过简单调节正负表面活性剂配比, 成功合成了 $\mathrm{BaWO}_{4} 、 \mathrm{BaMoO}_{4}$ 纳米带及分支状超结构 ${ }^{[225-126]}$, 如图 10 所示. 通过琼脂糖凝胶对离子扩散过程的影响, 调控了 $\mathrm{CaCO}_{3}$ 的结晶过程, 进而制备了 8 臂状 $\mathrm{CaCO}_{3}$ 分支结构 ${ }^{[127]}$.

扬州大学郭荣等人 ${ }^{[128]}$ 在十六烷基三甲基溴化铵 (CTAB) 和十二烷基苯甲磺酸钠(SDBS)的存在下, 合 成了 $\mathrm{Ag}$ 分支状结构, 实验结果表明, 阴阳离子表面 活性剂的比例及浓度对产物的形貌有重要的影响.

上海陶瓷研究所孙静等人 ${ }^{[1290}$ 以水合阱和 $\mathrm{Na}_{3} \mathrm{PO}_{4}$ 作为结构导向剂, 合成了在棒状主干上组装有片层 状结构的 $\beta-\mathrm{Co}(\mathrm{OH})_{2}$, 然后通过原位热分解制备了 $\mathrm{CO}_{3} \mathrm{O}_{4}$ 分支结构.

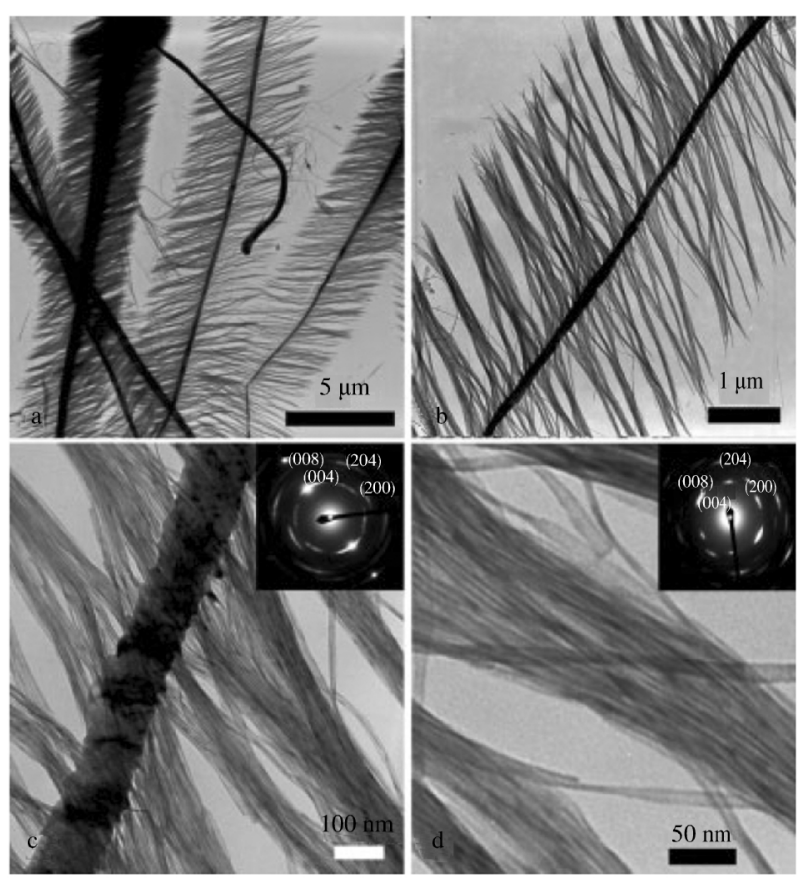

图 10 阴阳离子表面活性剂比例为 1.5 时制备 $\mathrm{BaMoO}_{4}$ 分 支结构的透射电镜照片 ${ }^{[126]}$

Fig.10 TEM images of the branched $\mathrm{BaMoO}_{4}$ nanostructures formed at the mixing ratio between anionic and cationic surfactants $(r)$ as $\mathbf{1 . 5}^{[126]}$ $\left[\mathrm{BaMoO}_{4}\right]=0.05 \mathrm{~mol} \cdot \mathrm{L}^{-1}, T=50{ }^{\circ} \mathrm{C}$; Insets in figures $\mathrm{c}, \mathrm{d}$ are the corresponding selected area electron diffraction (SAED) patterns. 


\section{4 其他结构}

除上述三种结构外,一些具有特定几何结构、尺 寸均一的纳米晶还可以作为纳米基元通过各种作用 力自组装形成复杂结构多级材料.一般来讲,纳米基 元的几何形状取决于材料的晶体结构,而基元的自 组装通过相邻纳米晶之间相对取向的自我调整或/ 和纳米晶界面间的晶格融合实现. 各组装基元具有 基本相同的晶体学取向. 比如, 如 $\mathrm{ZnO}$ 层状纳米结 构, $\mathrm{SnO}_{2}$ 空心八面体, 蒲公英状 $\mathrm{WO}_{3}$ 等 ${ }^{[130-132]}$.

新加坡国立大学的 Zeng 课题组 ${ }^{[133]}$ 制备了蒲公 英状的 $\mathrm{CuO}$ 结构, 如图 11 所示. 他们认为, 在水热 条件下, 溶液中首先生成了尺寸均一的 $\mathrm{CuO}$ 菱形纳 米晶, 为了降低体系能量, 各纳米片自发排列形成蒲 公英状 $\mathrm{CuO}$ 微球. 同样, 他们还利用金属锌在碱性 溶液中的水热反应通过改进的柯肯达尔效应获得了 空的蒲公英状的纳米结构 ${ }^{[134]}$. 这个过程没有应用任 何的辅助试剂, 主要依靠的是 $\mathrm{ZnO}$ 自身晶体生长的 各向异性得到的棒状结构组装在 $\mathrm{Zn}$ 核的周围而得 到, 这是一个模板辅助和利用晶体结构自范性生长 完美结合的例子.

2006 年, 中国科学技术大学谢毅课题组 ${ }^{[135]}$ 利用 $\mathrm{K}_{2} \mathrm{NaMoO}_{3} \mathrm{~F}_{3}$ 作为模板来制备 $\mathrm{MoS}_{2}$ 双层结构组成 的多级空心立方体盒子, 在此过程中, $\mathrm{MoS}_{2}$ 自身的 层状晶体结构特性对目标产物的形成起到了非常关 键的作用.

我们在 PVP 的存在下, 制备了盘状 $\gamma-\mathrm{MnO}_{2}$ 纳 米晶 ${ }^{[136]}$. 表征结果表明, 盘状集合体由尺寸相对均 一的纳米片组装而成. 六角形片层状是氢氧化镍的 一个典型结构, 最近, 我们还利用氢键相互作用, 将

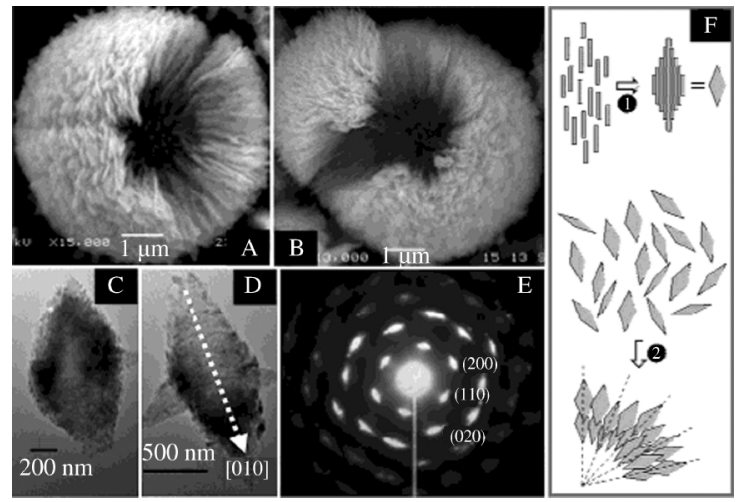

图 11 蒲公英状 $\mathrm{CuO}$ 的 $\operatorname{SEM}(\mathrm{A}, \mathrm{B})$ 和 $\operatorname{TEM}(\mathrm{C}, \mathrm{D})$ 图, (D)图的 SAED 图(E)照片和自组装示意图 $(F)^{[133]}$

Fig.11 SEM (A, B) and TEM $(C, D)$ images of dandelionlike CuO, SAED pattern shown in (D) (E), and the scheme illustrating the self-assembly process $(F)^{[133]}$
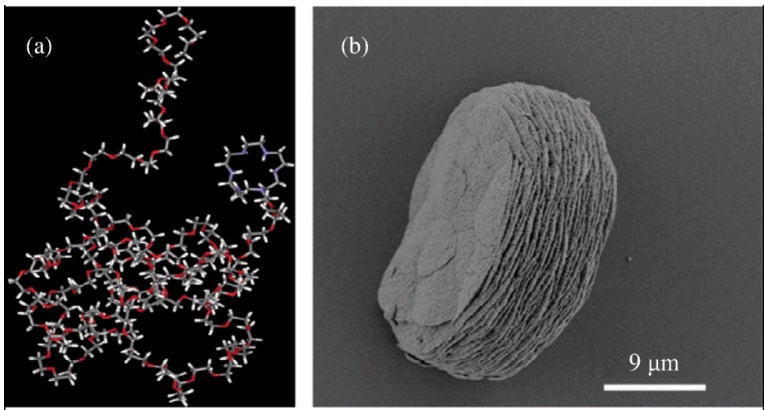

图 12 (a)嵌段共聚物模板分子的结构示意图, (b)多层结构 $\mathrm{CaCO}_{3}$ 的扫描电镜图片 [140]

Fig.12 (a) Structure model of the block polymer template, (b) scanning electron microscopy (SEM) images of the as-prepared $\mathrm{CaCO}_{3}$ with multi-layer structures $^{[140]}$

片层状前驱体组装为结构规整的拧三棱柱状 $\beta$ $\mathrm{Ni}(\mathrm{OH})_{2}$ 凹多面体 ${ }^{[137]}$.

通过巧妙地调节反应条件, 使 $\mathrm{PbSe}$ 纳米粒子 沿着不同的晶面取向连接, Murray 研究组 ${ }^{[138]}$ 得到了 包括线状、环状、锯齿状、锥状及枝状等在内的多种 纳米结构. 利用同样的原理, 新加坡国立大学 Zeng 等人得到了各种形状的 $\mathrm{CdS}$ 纳米环 ${ }^{[139]}$ 及 $\mathrm{SnO}_{2}$ 空心 八面体结构 ${ }^{[131]}$.

中国科技大学俞书宏课题组借助聚合物与无 机纳米晶之间的相互作用, 实现了多种纳米晶的分 级组装. 比如, 他们以双亲水性嵌段共聚物 (poly (ethylene gly-col)-b-poly(1,4,7,10,13,16-hexaazacyclooctadecan ethylene imine)作为晶体生长调控剂, 制备了 由多孔薄片自组装而成的多层结构方解石晶体(如 图 12 所示), 并从理论上模拟了晶体-聚合物之间的 相互作用 ${ }^{[140]}$. 采用磷酸化双亲性嵌段共聚物调控无 机晶体生长, 首次在 $\mathrm{BaCO}_{3}$ 矿物质膜上合成了新颖 的双螺旋及其柱体螺旋纳米线 ${ }^{[141]}$; 在阳离子表面活 性剂 CTAB 的协同作用下, 利用简单的低温溶液反 应, 将多面体纳米晶体进行取向搭接, 成功获得了具 有螺旋状棒形等级结构的铇铅矿微晶, 并对其光学 性质进行了研究 ${ }^{[142]}$. 同样在 CTAB 的协同作用下, 他们还将 $\mathrm{MnWO}_{4}$ 纳米棒组装为海胆状微米球, 并 探讨了 $\mathrm{pH}$ 值及反应温度对产物形貌的影响 ${ }^{[143]}$.

\section{5 总结与展望}

从国际纳米材料设计和研究的发展趋势来看, 随着对纳米材料与纳米结构研究工作的不断深人, 研究正逐步由广泛探索向具有重要应用背景的纳米 材料和重要的深层次科学问题方向转移,纳米材料 
的制备研究工作逐步向高精度可控制备、制备方法 原理化和规律化、纳米结构构筑不断复杂化、功能化 集成与复合等方向转移. 分级结构材料除了具有纳 米结构单元组合产生的耦合效应、协同效应等而备 受关注, 更重要的是, 为进一步的新型功能器件的构 筑提供了坚实的基础, 这使其成为目前国际上一个 重要的前沿研究领域.

分级结构材料的构筑主要涉及次级结构材料在 初级结构材料上的异质成核生长或两种材料之间的 成分交换. 本文通过对分级结构材料的构筑原理和 应用实例的介绍, 展示了目前分级结构材料的液相 合成策略. 虽然分级结构的构筑已取得了不少成功 的实例, 然而由于合成体系的复杂性, 最终的自组装 过程对很多实验参数都比较敏感, 因此对其调控也 相对比较复杂, 目前该方面的研究仍处于初级阶段. 主要体现在对分级结构的合成在很大程度上仍具有 随机性, 对影响纳米基元组装过程的关键因素缺乏 规律性的认识, 多数情况下尚无法实现精确的可控 组装. 另外, 对于材料组装引起的性质变化尚缺乏深 刻认识. 这些都是分级结构材料走向实用化的基础 和关键. 总的来讲, 分级结构材料的研究具有很大 的发展潜力. 对分级结构材料的探索研究将为纳米 科技的发展注人新的活力.

\section{References}

1 Nudelman, F.; Gotliv, B. A.; Addadi, L.; Weiner, S. J. Struct. Biol., 2006, 153: 176

2 Feng, L.; Li, S.; Li, Y.; Li, H. J.; Zhang, L. J.; Zhai, J.; Song, Y. L.; Liu, B. Q.; Jiang, L.; Zhu, D. B. Adv Mater., 2002, 14: 1857

3 Choi, S. J.; Suh, K. Y.; Lee, H. H. J. Am. Chem. Soc., 2008, 130: 6312

4 Fang, B. Z.; Kim, M.; Kim, J. H.; Yu, J. S. Langmuir, 2008, 24: 12068

5 Yang, R.; Chueh, Y. L.; Morber, J. R.; Snyder, R.; Chou, L. J.; Wang, Z. L. Nano Lett., 2007, 7: 269

6 Lyon, J. L.; Fleming, D. A. Nano Lett., 2004, 4: 719

7 Xu, Z. C.; Sun, S. H. J. Am. Chem. Soc., 2007, 129: 8698

8 Nash, M. A.; Lai, J. J.; Hoffman, A. S.; Yager, P.; Stayton, P. S. Nano Lett., 2010, 10: 85

9 Li, J. J.; Wang, Y. A.; Guo, W. Z.; Keay, J. C.; Mishima, T. D.; Johnson, M. B.; Peng, X. G. J. Am. Chem. Soc., 2003, 125: 12567

10 Pan, D. C.; Wang, Q.; Jiang, S. C.; Ji, X. L.; An, L. J. Adv. Mater., 2005, 17: 176

11 Li, L.; Protiere, M.; Reiss, P. Chem. Mater., 2008, 20: 2621

12 Dubertret, B.; Skourides, P.; Norris, D. J.; Noireaux, V.; Brivanlou, A. H.; Libchaber, A. Science, 2002, 298: 1759
13 Han, M. Y.; Gao, X. H.; Su, J. Z.; Nie, S. M. Nature Biotech., 2001, 19: 631

14 Chan, W. C. W.; Nie, S. M. Science, 1998, 281: 2016

15 Nann, T.; Mulvaney, P. Angew. Chem. Int. Edit., 2004, 43: 5393

16 Li, H. B.; Li, Y. L.; Cheng, J. Chem. Mater., 2010, 22: 2451

17 Jing, L.H.; Yang, C. H.; Qiao, R. R.; Niu, M.; Du, M. H.; Wang, D. Y.; Gao, M. Y.Chem. Mater., 2010, 22: 420

18 Bruchez, M.; Moronne, M.; Gin, P.; Weiss, S.; Alivisatos, A. P. Science, 1998, 281: 2013

19 Donath, E.; Sukhorukov, G. B.; Caruso, F.; Davis, S. A.; Möhwald, H. Angew. Chem. Int. Edit., 1998, 37: 2201

20 Caruso, F.; Lichtenfeld, H.; Giersig, M.; Möhwald, H. J. Am. Chem. Soc., 1998, 120: 8523

21 Caruso, F.; Caruso, R. A.; Möhwald, H. Science, 1998, 282: 1111

22 Caruso, R. A.; Susha, A.; Caruso, F. Chem. Mater., 2001, 13: 400

23 Shin, W. J.; Kim, J. Y.; Chob, G.; Lee, J. S. J. Mater. Chem., 2009, 19: 7322

24 Liu, J. G.; Liang, J. G.; Han, H. Y.; Sheng, Z. H. Mater. Lett., 2009, 63: 2224

25 Lu, Y.; Yin, Y. D.; Xia, Y. N. Adv. Mater., 2001, 13: 271

26 Cho, E. C.; Camargo, P. H. C.; Xia, Y. N. Adv. Mater., 2010, 22 : 744

27 Qiu, P. H.; Mao, C. B. ACS Nano, 2010, 4: 1573

28 Ge, C.; Zhang, D. Z.; Wang, A. L.; Yin, H. B.; Ren, M.; Liu, Y. M.; Jiang, T. S.; Yu, L. B. J. Phys. Chem. Solid., 2009, 70: 1432

29 Lee, C.; Kim, I.; Shin, H.; Kim, S.; Cho, J. Nanotechnology, 2010, 21: 185704

30 Yeom, B.; Char, K. Chem. Mater., 2010, 22: 101

31 Protiere, M.; Reiss, P. Small, 2007, 3: 399

32 Reiss, P.; Protiere, M.; Li, L. Small, 2009, 5: 154 and the literature therein

33 Carbone, L.; Nobile, C.; Giorg, M. D.; Sala, F. D.; Morello, G.; Pompa, P.; Hytch, M.; Snoeck, E.; Fiore, A.; Franchini, I. R.; Nadasan, M.; Silvestre, A. F.; Chiodo, L.; Kudera, S.; Cingolani, R.; Krahne, R.; Manna, L. Nano Lett., 2007, 7: 2942

34 Aharoni, A.; Mokari, T.; Popov, I.; Banin, U. J. Am. Chem. Soc., 2006, 128: 257

35 Liu, H. T.; Owen, J. S.; Alivisatos, A. P. J. Am. Chem. Soc., 2007, 129: 305

36 Blackman, B.; Battaglia, D.; Peng, X. G. Chem. Mater., 2008, 20 4847

37 Chin, P. T. K.; Donega, C. D. M.; Bavel, S. S.; Meskers, S. C. J.; Sommerdijk, N.; Janssen, R. A. J. J. Am. Chem. Soc., 2007, 129: 4880

38 Kim, S.; Fisher, B.; Eisler, H. J.; Bawendi, M. J. Am. Chem. Soc., 2003, 125: 11466

39 Zimmer, J. P.; Kim, S. W.; Ohnishi, S.; Tanaka, E.; Frangioni, J. V.; Bawendi, M. G. J. Am. Chem. Soc., 2006, 128: 2526

40 Sung, Y. M.; Park, K. S.; Lee, Y. J.; Kim, T. G. J. Phys. Chem. C, 2007, 111: 1239

41 Habas, S. E.; Lee, H.; Radmilovic, V.; Somorjai, G. A.; Yang, P. D. 
Nature Mater, 2007, 6: 692

42 Fan, F. R.; Liu, D. Y.; Wu, Y.; Duan, S.; Xie, Z. X.; Jiang, Z. Y.; Tian, Z. Q. J. Am. Chem. Soc., 2008, 130: 6949

43 Ge, C.; Zhang, D. Z.; Wang, A. L.; Yin, H. B.; Ren, M.; Liu, Y. M.; Jiang, T. S.; Yu, L. B. J. Am. Chem. Soc., 2010, 132: 2506

44 Min, M.; Kim, C.; Yang, Y. I.; Yi, J.; Lee, H. Phys. Chem. Chem. Phys., 2009, 11: 9759

45 Alayoglu, S.; Zavalij, P.; Eichhorn, B. ACS Nano, 2009, 3: 3127

46 Wu, Y.; Jiang, P.; Jiang, M.; Wang, T. W.; Guo, C. F.; Xie, S. S.; Wang, Z. L. Nanotechnology, 2009, 20: 305602

47 Chen, Y. J.; Xue, X. Y.; Wang, T. H. Nanotechnology, 2005, 16 : 1978

48 Krishna, K. S.; Vivekanandan, G.; Ravindera, D.; Eswaramoorthy, M. Chem. Commun., 2010, 46: 2989

49 Teng, X. W.; Black, D.; Watkins, N. J.; Gao, Y. L.; Yang, H. Nano Lett., 2003, 3: 261

50 Li, X. L.; Lou, T. J.; Sun, X. M.; Li, Y. D. Inorg. Chem., 2004, 43: 5442

51 Bao, J. C.; Liang, Y. Y.; Xu, Z.; Si, L. Adv. Mater., 2003, 15: 1832

52 Yin, Y. D.; Rioux, R. M.; Erdonmez, C. K.; Hughes, S.; Somorjai, G. A.; Alivisatos, A. P. Science, 2004, 30: 711

53 Kim, S. H.; Yin, Y. D.; Alivisatos, A. P.; Somorjai G. A.; Yates J. T. J. Am. Chem. Soc., 2007, 129: 9510

54 Gao, J. H.; Liang, G. L.; Zhang, B.; Kuang, Y.; Zhang X. X.; Xu, B. J. Am. Chem. Soc., 2007, 129: 1428

55 Wang, Y. L.; Cai, L.; Xia, Y. N. Adv. Mater., 2005, 17: 473

56 Jiao, S. H.; Xu, L. F.; Jiang, K.; Xu, D. S. Adv. Mater., 2006, 18: 1174

57 Gao, J. H.; Zhang, B.; Zhang, X. X.; Xu, B. Angew. Chem. Int. Edit., 2006, 45: 1220

58 Fan, H. J.; Knez, M.; Scholz, R.; Nielsch, K.; Pippel, E.; Hesse, D.; Gösele, U.; Zacharias, M. Nanotechnology, 2006, 17: 5157

59 Fan, H. J.; Knez, M.; Scholz, R.; Nielsch, K.; Pippel, E.; Hesse, D.; Zacharias, M.; Gosele, U. Nature Mater., 2006, 5: 627

60 Railsback, J. G.; Johnston-Peck, A. C.; Wang, J. W.; Tracy, J. B. ACS Nano, 2010, 4: 1913

61 Tian, L.; Yang, X. F.; Lu, P.; Williams, I. D.; Wang, C. H.; Ou, S. Y.; Liang, C. L.; Wu, M. M. Inorg. Chem., 2008, 47: 5522

62 Gao, P. X.; Wang, Z. L. J. Am. Chem. Soc., 2003, 125: 11299

63 Wang, Q.; Geng, B. Y.; Wang, S. Z.; Ye, Y. X.; Tao, B. Chem. Commun., 2010, 46: 1899

64 Shibata, T.; Bunker, B. A.; Zhang, Z.; Meisel, D.; Vardeman, C. F.; Gezelter, J. D. J. Am. Chem. Soc., 2002, 124: 11989

65 Chen, J.; Wiley, B.; McLellan, J. M.; Xiong, Y.; Li, Z. Y.; Xia, Y. N. Nano Lett., 2005, 5: 2058

66 Cobley, C. M.; Campbell, D. J.; Xia, Y. N. Adv. Mater., 2008, 20 748

67 Sun, Y.; Mayers, B.; Xia, Y. N. Nano Lett., 2002, 2: 481

68 Sun, Y.; Mayers, B.; Xia, Y. N. Adv. Mater., 2003, 15: 641

69 Chen, J.; McLellan, J. M.; Siekkinen, A.; Xiong, Y.; Li, Z. Y.; Xia, Y. N. J. Am. Chem. Soc., 2006, 128: 14776
70 Sun, Y.; Wiley, B. J.; Li, Z. Y.; Xia, Y. N. J. Am. Chem. Soc., 2004, 126: 9399

71 Lu, X.; Tuan, H. Y.; Chen, J.; Li, Z. Y.; Korgel, B. A.; Xia, Y. N. J. Am. Chem. Soc., 2007, 129: 1733

72 Lee, W. R.; Kim, M. G.; Choi, J. R.; Park, J. I.; Ko, S. J.; Oh, S. J.; Cheon, J. J. Am. Chem. Soc., 2005, 127: 16090

73 Park, J. I.; Lee, W. R.; Jun, Y. W.; Lee, J. S.; Lee, W. R.; Cheon, J. J. Am. Chem. Soc., 2004, 126: 9072

74 Chen, D.; Li, J. J.; Shi, C. S.; Du, X. W.; Zhao, N. Q.; Sheng, J.; Liu, S. Chem. Mater., 2007, 19: 3399

75 Lu, Y.; Zhao, Y.; Yu, L.; Dong, L.; Shi, C.; Hu, M. J.; Xu, Y. J.; Wen, L. P.; Yu, S. H. Adv. Mater., 2010, 22: 1407

76 Achermann, M.; Petruska, M. A.; Kos, S.; Smith, D. L.; Koleske, D. D.; Klimov, V. I. Nature, 2004, 429: 642

77 Friedman, R. S.; McAlpine, M. C.; Ricketts, D. S.; Ham, D.; Lieber, C. M. Nature, 2005, 434: 1085

78 Li, D. Y.; Wu, Y.; Fan, R.; Yang, P. D.; Majumdar, A. Appl. Phys. Lett., 2003, 83: 3186

79 Gudiksen, M. S.; Lauhon, L. J.; Wang, J. F.; Smith, D. C.; Lieber, C. M. Nature, 2002, 415: 617

80 Yan, R. X.; Gargas, D.; Yang, P. D. Nature Photonics, 2009, 3: 569

81 Penn, S. G.; He, L.; Natan, M. J. Curr. Opin. Chem. Biol., 2003, 7: 609

82 Jung, J.; Seo, D.; Park, G.; Ryu, S.; Song, H. J. Phys. Chem. C, 2010, 114: 12529

83 Salem, A. K.; Searson, P. C.; Leong, K. W. Nat. Mater., 2003, 2: 668

84 Liu, F.; Lee, J. Y.; Zhou, W. J. Small, 2006, 2: 121

85 Mokari, T.; Rothenberg, E.; Popov, I.; Costi, R.; Banin, U. Science, 2004, 304: 1787

86 Saunders, A. E.; Popov, I.; Banin, U. J. Phys. Chem. B, 2006, 110: 25421

87 Menagen, G.; Mocatta, D.; Salant, A.; Popov, I.; Dorfs, D.; Banin, U. Chem. Mater., 2008, 20: 6900

88 Mokarim, T.; Sztrumm, C. G.; Salantm, A.; Rabanim, E.; Baninm, U. Nature Mater., 2005, 4: 855

89 Yu, H.; Chen, M.; Rice, P. M.; Wang, S. X.; White, R. L.; Sun, S. H. Nano Lett., 2005, 5: 379

90 Carbone, L.; Kudera, S.; Giannini, C.; Ciccarella, G.; Cingolani, R.; Cozzoli, P. D.; Manna, L. J. Mater. Chem., 2006, 16: 3952

91 Huang, S. S.; Huang, J. M.; Yang, J. A.; Peng, J. J.; Zhang, Q. B.; Peng, F.; Wang, H. J.; Yu, H. Chem.-Eur. J., 2010, 16: 5920

92 Hewa-Kasakarage, N. N.; Kirsanova, M.; Nemchinov, A.; Schmall, N.; El-Khoury, P. Z.; Tarnovsky, A. N.; Zamkov, M. J. Am. Chem. Soc., 2009. 131: 1328

93 Menagen, G.; MacDonald, J. E.; Shemesh, Y.; Popov, I.; Banin, U. J. Am. Chem. Soc., 2009, 131: 17406

94 Talapin, D. V.; Shevchenko, E. V.; Murray, C. B.; Kornowski, A.; Förster, S.; Weller, H. J. Am. Chem. Soc., 2004, 126: 12984

95 Franchini, I. R.; Bertoni, G.; Falqui, A.; Giannini, C.; Wang, L. 
W.; Manna, L. J. Mater. Chem., 2010, 20: 1357

96 Lakshmi, B. B.; Patrissi, C. J.; Martin, C. R. Chem. Mater., 1997, 9: 2544

97 Li, Y.; Cheng, G. S.; Zhang, L. D. J. Mater. Res., 2000, 15: 2305

98 Nakamura, H.; Matsui, Y. J. Am. Chem. Soc., 1995, 117: 2651

99 Cao, H. Q.; Xu, Y.; Hong, J. M.; Liu, H. B.; Yin, G.; Li, B. L.; Tie, C. Y.; Xu, Z. Adv. Mater., 2001, 13: 1393

100 Limmer, S. J.; Seraji, S.; Forbess, M. J.; Wu, Y.; Chou, T. P.; Nguyen, C.; Cao, G. Z. Adv Mater., 2001, 13: 1269

101 Nicewarner-Pen, S. R.; Freeman, R. G.; Reiss, B. D.; He, L.; Pen, D. J.; Walton, I. D.; Cromer, R.; Keating, C. D.; Natan, M. J. Science, 2001, 294: 137

102 Liang, H. P.; Guo, Y. G.; Hu, J. S.; Zhu, C. F.; Wan, L. J.; Bai, C. L. Inorg. Chem., 2005, 44: 3013

103 Chen, M.; Searson, P. C.; Chien, C. L. J. Appl. Phys., 2003, 93 : 8253

104 Salem, A. K.; Chen, M.; Hayden, J.; Leong, K. W.; Searson, P. C. Nano Lett., 2004, 4: 1163

105 Pena, D. J.; Mbindyo, J. K. N.; Carado, A. J.; Mallouk, T. E.; Keating, C. D.; Razavi, B.; Mayer, T. S. J. Phys. Chem. B, 2002, 106: 7458

106 Guo, Y. G.; Wan, L. J.; Zhu, C. F.; Yang, D. L.; Chen, D. M.; Bai, C. L. Chem. Mater., 2003, 15: 664

107 Son, D. H.; Hughes, S. M.; Yin, Y. D.; Alivisatos, A. P. Science, 2004, 306: 1009

108 Robinson, R. D.; Sadtler, B.; Demchenko, D. O.; Erdonmez, C. K.; Wang, L. W.; Alivisatos, A. P. Science, 2007, 317: 355

109 Demchenko, D. O.; Robinson, R. D.; Sadtler, B.; Erdonmez, C. K.; Alivisatos, A. P.; Wang, L. W. ACS Nano, 2008, 2: 627

110 Luther, J. M.; Zheng, H.; Sadtler, B.; Alivisatos, A. P. J. Am. Chem. Soc., 2009, 131: 16851

111 Wark, S. E.; Hsia, C. H.; Son, D. H. J. Am. Chem. Soc., 2008, 130: 9550

112 Kovalenko, M. V.; Talapin, D. V.; Loi, M. A.; Cordella, F.; Hesser, G.; Bodnarchuk, M. I.; Heiss, W. Angew. Chem. Int. Edit., 2008, 47: 3029

113 Gao, X. P.; Zheng, Z. F.; Zhu, H. Y.; Pan, G. L.; Bao, J. L.; Wu, F.; Song, D. Y. Chem. Commun., 2004: 1428

114 Zhang, D. F.; Sun, L. D.; Zhang, J.; Yan, Z. G.; Yan, C. H. Crystal Growth \& Design, 2008, 8: 3609

115 Zhang, T.; Dong, W. J.; Keeter-Brewer, M.; Konar, S.; Njabon, R. N.; Tian, Z. R. J. Am. Chem. Soc., 2006, 128: 10960

116 Sounart, T. L.; Liu, J.; Voigt, J. A.; Hsu, J. W. P.; Spoerke, E. D.; Tian, Z. R.; Jiang, Y. B. Adv. Funct. Mater., 2006, 16: 335

117 Sounart, T. L.; Liu, J.; Voigt, J. A.; Huo, M.; Spoerke, E. D.;
McKenzie, B. J. Am. Chem. Soc., 2007, 129: 15786

118 Lao, J. Y.; Wen, J. G.; Ren, Z. F. Nano Lett., 2002, 2: 1287

119 Dick, K. A.; Deppert, K.; Larsson, M. W.; Martensson, T.; Seifert, W.; Wallenberg, L. R.; Samuelson, L. Nat. Mater., 2004, 3: 380

120 Milliron, D. J.; Hughes, S. M.; Cui, Y.; Mannal, L.; Li, J. B.; Wang, L. W.; Alivisatos, A. P. Nature, 2004, 430: 190

121 Yang, H. G.; Zeng, H. C. J. Phys. Chem. B, 2004, 108: 819

122 Yang, H. G.; Zeng, H. C. J. Am. Chem. Soc., 2005, 127: 270

123 Zhang, D. F.; Su, L. D.; Jia, C. J.; Yan, Z. G.; Yan, C. H. J. Am. Chem. Soc., 2005, 127: 13492

124 Shi, H. T.; Qi, L. M.; Ma, J. M.; Cheng, H. M. J. Am. Chem. Soc., 2003, 125: 3450

125 Shi, H. T.; Qi, L. M.; Ma, J. M.; Cheng, H. M.; Zhu, B. Y. Adv. Mater., 2003, 15: 1647

126 Shi, H. T.; Qi, L. M.; Ma, J. M.; Wu, N. Z. Adv. Funct. Mater., 2005, 15: 442

127 Yang, D.; Qi, L. M.; Ma, J. M. Chem. Commun., 2003: 1180

128 Fan, L.; Guo, R. Crystal Growth \& Design, 2008, 8: 2150

129 Shao, Y. Z.; Sun, J.; Gao, L. J. Phys. Chem. C, 2009, 113: 6566

130 Wu, J. J.; Wen, H. I.; Tseng, C. H.; Liu, S. C. Adv. Funct. Mater., 2004, 14: 806

131 Yang, H. G.; Zeng, H. C. Angew. Chem. Int. Edit., 2004, 43: 5930

132 Gu, Z. J.; Zhai, T. Y.; Gao, B. F.; Sheng, X. H.; Wang, Y. B.; Fu, H. B.; Ma, Y.; Yao, J. N. J. Phys. Chem. B, 2006, 110: 23829

133 Liu, B.; Zeng, H. C. J. Am. Chem. Soc., 2004, 126: 8124

134 Liu, B.; Zeng, H. C. J. Am. Chem. Soc., 2004, 126: 16744

135 Ye, L. N.; Wu, C. Z.; Guo, W.; Xie, Y. Chem. Commun., 2006: 4738

136 Wang, N.; Cao, X.; He, L.; Zhang, W.; Guo, L.; Chen, C. P.; Wang, R. M.; Yang, S. H. J. Phys. Chem. C, 2008, 112: 365

137 Zhou, W.; Yao, M.; Guo, L.; Li, Y. M.; Li, J. H.; Yang, S. H. J. Am. Chem. Soc., 2009, 131: 2959

138 Cho, K. S.; Talapin, D. V.; Gaschler, W.; Murray, C. B. J. Am. Chem. Soc., 2005, 127: 7140

139 Liu, B.; Zeng, H. C. J. Am. Chem. Soc., 2005, 127: 18262

140 Chen, S. F.; Yu, S. H.; Wang, T. X.; Jiang, J.; Colfen, H.; Hu, B.; Yu, B. Adv. Mater., 2005, 17: 1461

141 Zhu, J. H.; Yu, S. H.; Xu, A. W.; Colfen, H. Chem. Commun., 2009: 1106

142 Liu, B.; Yu, S. H.; Li, L. J.; Zhang, Q.; Zhang, F.; Jiang, K. Angew. Chem. Int. Edit., 2004, 43: 4745

143 Zhou, Y. X.; Zhang, Q.; Gong, J. Y.; Yu, S. H. J. Phys. Chem. C, 2008, 112: 13383 